\title{
Postoptimization of the model of water supply for urban and industrial agglomeration
}

\author{
Jacek Wawrzosek ${ }^{1}$, and Szymon Ignaciuk $^{1^{*}}$ \\ ${ }^{1}$ Department of Applied Mathematics and Computer Science, Faculty of Production Engineering, University of Life Sciences in \\ Lublin, Głeboka 28, PL 20612 Lublin, Poland
}

\begin{abstract}
A case study of the tools used by an analyst of the economic aspects of the operation of the water supply network has been undertaken in this paper. All issues discussed here are formulated by using degenerated linear programming models $(P L)$. Below, it is noted that the linear dependence of binding constraints ( $C O$ ) distorts standard postoptimization procedures in $P L$. This observed fact makes postoptimization analysis mostly unhelpful for an average analyst due to problems with the interpretation of ambiguous sensitivity reports which are obtained from popular computer packages. In standard postoptimization methods, changes to single parameters of the right-hand vector $C O$ are analyzed or referred to parametric linear programming that unfortunately requires prior knowledge of mathematically and economically justified vectors of changes of right-hand sides $C O$. Therefore, it is suggested that modifications are introduced to some of the postoptimization procedures in this work. For issues in the field of hydrology, the following were presented: interpretation and methods of generating justified vectors of changes of right-hand sides of limiting conditions. And so, the procedure of generating infinitely many solutions of the dual issue based on certain vectors orthogonal to the vector of right-hand sides of constraint conditions was demonstrated. Furthermore, the same orthogonal vectors were used to obtain nodal solutions of the dua01 model and the corresponding vectors of changes of the entire righthand sides of the constraint conditions. Then, managerial interpretation was applied to this way of proceeding. The methods presented in the work serve to improve the functioning of the system of water supply.
\end{abstract}

Keywords: linear programming, sensitivity analysis, postoptimization, water supply in agglomerations

\section{Introduction}

Considering the flow volume, the Vistula River is an almost unlimited source of water for all potential users. 76 cities and towns are located on its banks. In Silesian Province, the dammed Czerniański Reservoir, created for retention purposes and as a potable water reservoir at the site of the Biała and Czarna Wisełka confluence in Wisła Czarne, is already operated by Wodociagi Ziemi Cieszyńskiej Sp. z o.o. in Ustroń - a municipal enterprise whose shareholders are 11 municipalities, i.e. Cieszyn, Ustroń, Skoczów, Wisła, Strumień, Chybie, Goleszów, Dębowiec, Hażlach, Brenna and Istebna. The water produced in the Water Treatment Station Wisła Czarne supplies the water supply network by providing drinking water to consumers in the above-mentioned cities and towns. On average, the station produces around 8400 $\left[\mathrm{m}^{3} /\right.$ day] water, and its actual production capacity is limited by the water permit, which allows for sourcing up to $12960\left[\mathrm{~m}^{3} /\right.$ day] of raw water from the intake.

However, the water in the Vistula is currently still of poor quality and therefore users are reluctant to use it. Hence, in the system which supplies Kraków with water, there are four treatment stations, and the water is sourced from the rivers Sanka, Dłubnia, Rudawa and from the Dobczycki Reservoir on the Rabie River.

Nevertheless, there are several significant water intakes on the Vistula in the Warsaw section. Hence, $70 \%$ of the Warsaw agglomeration's demand for water is satisfied by the Vistula [1]

In order to meet the demand for water for municipal and industrial purposes, the city of Płock uses both the surface resources from the Vistula River and underground ones from deep wells. At the same time, the Vistula River has approx. a 55\% share in the total amount of water supplied to the recipients in this city. Still in the spring of 2015, the scientists from the International Institute of the European Regional Centre for Ecohydrology PAN sounded alarm in the media, claiming that Poland is in danger of drought and that Poland's energy security and

Corresponding author: szymon.ignaciuk@up.lublin.pl 
systematic power supply are directly dependent on water management. The Kozienice power plant produces approx. $13 \%$ of electricity in Poland, including its provision for the residents of Warsaw and Mazovia. We remember how in the summer of 2015 the menace of limiting domestic industrial production began to take a real form because the blocks in the Kozienice power plant are cooled with water from the Vistula. At high water temperatures in the Vistula reaching 25 degrees $\mathrm{C}$ and low water level in the river the government decided that periodic restrictions will be imposed on supply and power consumption for large consumers. This decision secured the energy system in a situation of a prolonged heat wave. At that time, the water in the Vistula reached an average record low of between 41 and 50 centimetres. In the localities where problems arose, local authorities introduced restrictions on water supply from the water supply system and bans on e.g. watering gardens, cleaning vehicles, filling swimming pools using waterworks. The owners of horticultural farms found themselves in a dramatic situation, requiring large amounts of water for production. An unpleasant situation also prevailed on the reservoir "Mylof" on the Brda River in Chojnice district. The water level reached $23 \mathrm{~cm}$ below the minimum level of accumulation. The "Mylof" hydro power plant practically did not work, and the low water level threatened the largest trout farm in the country. Possibilities of reaction to such a situation tend to be limited. At the same time, low water levels appeared not only because of high temperatures, but as a result of the dropping groundwater level. As the next cause, the recently relatively light winters are mentioned, without much snowfall and strong frosts. As a result, water usually flows into the rivers, with smaller amounts soaking into the ground. In the described period there were warnings that a rapid onset of deep frosts after a period of drought, when the rivers have not been replenished with water, may cause freezing of rivers and will cause the necessity of switching off powergenerating units and a lack of water for municipal use. Thus, it can be seen that efficient water management in agricultural and forest areas as well as for the needs of urban-industrial agglomerations should be perceived as one of the key issues of contemporary planning [2].

Making optimal decisions in this area requires the use of advanced methods and models of operational research. In particular, management decisions upon hydrological problems are repeatedly based on linear programming models. The above-mentioned variability of parameters in the developed mathematical models requires the use of postoptimization procedures. However, despite the interest of various authors from many fields, postoptimization for degenerated models has so far failed to find proper mathematical and managerial methods in literature [3-10]. This work presents the possibilities of using new, extended methods of inference within the postoptimization already based on classically obtained tables of the simplex method. Hence, this work is a development of postoptimization techniques for the degenerated problems of linear programming undertaken in [2, 11-13] on hydrological problems. The following case study was presented to show the mathematical and interpretative difficulties appearing here and new methods of solving them.

\section{Postoptimization analysis in hydrology}

Let us assume that water is collected in an agglomeration from $p=2$ water intakes. Because water distribution is variable, the amount of water taken from intakes No. 1 and 2 are decision variables, respectively $x_{1}$ and $x_{2}$, represented by the vector $\boldsymbol{x}^{T}=\left[x_{1} ; x_{2}\right]$, and periodically determined in different units, e.g. $\left[d m^{3} \cdot s^{-1}\right]$, $\left[d m^{3} \cdot h^{-1}\right], \quad\left[m^{3} \cdot d^{-1}\right], \quad\left[m^{3} \cdot\right.$ month $\left.^{-1}\right]$, $\left[\mathrm{m}^{3} \cdot\right.$ year $\left.^{-1}\right]$. The current periodic demand for water of an agglomeration is variable and amounts to at least 200 units (CO1). In addition, there are restrictions due to the principle of the inviolability of water flow in the water gauge cross-section "S" and technical reasons of the dependence between intakes (CO2, CO3 CO4). By recording the total cost of water intake from two intakes in the form of a minimized objective function (OF: $\boldsymbol{c} \cdot \boldsymbol{x} \rightarrow \min )$, with the vector of cost factors in the form of $c=\left[c_{1} ; c_{2}\right]=[2 ; 3]$ the model $\boldsymbol{M 1}$ of a linear form programming was obtained and studied further:

Objective Function $(O F): O F(\boldsymbol{x})=\boldsymbol{c} \cdot \boldsymbol{x} \rightarrow \min$. Constraints $(\mathrm{CO})$ :

$\begin{array}{ll}\text { CO1: } & x_{1}+x_{2} \geq 200, \\ \text { CO2: } & x_{1}+2 x_{2} \geq 280, \\ \text { CO3: } & 4 x_{1}+3 x_{2} \leq 720, \\ \text { CO4: } & 4 x_{1}+7 x_{2} \geq 1040 .\end{array}$

Boundary Restrictions $(B R)$ : $\quad x_{1}, x_{2} \geq 0$.

Many software packages (like Matlab, Excel. Lindo, WinQSB, Storm, CPLEX, OSL, PC-Prog or XMP [11, 14]) include linear programming solvers nowadays. By using one of them, the optimal solution to the $\boldsymbol{M} \mathbf{1}$ model in the form of $\boldsymbol{x}^{*}=[120 ; 80]^{T}$ was obtained (see tab. 1). Because, however, each of $n=4$ constraints per two variables is met with equality for $\boldsymbol{x}=\boldsymbol{x}^{*}$ (compare columns The final value and The right-hand side of the constraints) thus a degenerate problem of linear programming with an infinite number of dual model solutions to $\boldsymbol{M 1}$ has been obtained (model $\boldsymbol{D} \_\boldsymbol{M 1}$ ). Because the number of the constraints $\boldsymbol{M 1}$ and therefore the number of decision variables in the dual model is $n=4$ and all $C O$ for $\boldsymbol{M 1}$ are binding and the row of matrix formed from the coefficient of binding conditions (here the entire array of left-hand sides of $C O$, i.e. $A$ ) is equal to 2 , so the set of solutions $P$ in the dual model is a subset of the dimension space:

$$
n-r(A)=2 .
$$

This results from the theorem of complementarity $[15$, p. 136] and from the Kronecker-Capelli theorem.

Until now, a simple description of the set $P$ with degeneration has not been presented in the literature. Naturally, the vector of dual prices $y_{0}^{*}=[1 ; 1 ; 0 ; 0]^{T}$, i.e. $y_{0}^{*} \in P$ generated in the report belongs to the set, but this is only one point of the two-dimensional set. In [13], it was proposed to find vectors spanning the space containing $P$ on the basis of the differences between several vectors taken from alternative base solutions $\boldsymbol{M 1}$. 
Because the generation of many alternative solutions is difficult, this article simplifies this postoptimization stage by designating vectors spanning the space of the dual model solutions using directly the last simplex table generated for the model $\boldsymbol{M 1}$ (see Tab. 2).

Table 1. Sensitivity report generated by Excel for the model $\mathbf{M 1}$.

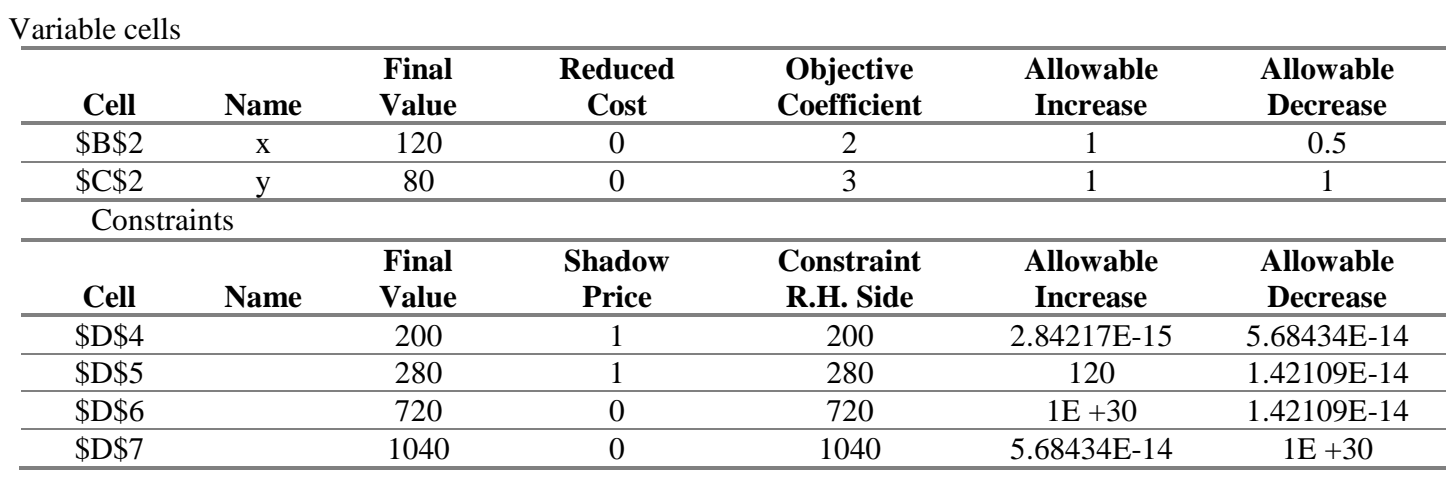

Table 2. The matrix columns $B^{-1}$ against the background of the last simplex table for the model $\mathbf{M 1}$.

\begin{tabular}{|c|c|c|c|c|c|c|c|c|c|c|c|}
\hline \multicolumn{2}{|c|}{$\mathrm{c}_{\mathrm{i}}$} & 2 & 3 & 0 & 0 & 0 & 0 & $\mathrm{M}$ & $\mathrm{M}$ & $\mathrm{M}$ & \\
\hline $\boldsymbol{c}_{\mathrm{B}}$ & $\boldsymbol{x}_{\mathrm{B}}$ & $\mathrm{x}_{1}$ & $\mathrm{x}_{2}$ & $\mathrm{x}_{3}$ & $\mathrm{x}_{4}$ & $\mathrm{x}_{5}$ & $\mathrm{x}_{6}$ & $\mathrm{x}_{7}$ & $\mathrm{x}_{8}$ & $\mathrm{x}_{9}$ & $b$ \\
\hline 2 & $\mathrm{x}_{1}$ & 1 & 0 & -2.33 & 0 & 0 & 0.33 & 2.33 & 0 & -0.33 & 120 \\
\hline 3 & $\mathrm{x}_{2}$ & 0 & 1 & 1.33 & 0 & 0 & -0.33 & -1.33 & 0 & 0.33 & 80 \\
\hline 0 & $\mathrm{x}_{5}$ & 0 & 0 & 5.33 & 0 & 1 & -0.33 & -5.33 & 0 & 0.33 & 0 \\
\hline 0 & $\mathrm{x}_{4}$ & 0 & 0 & 0.33 & 1 & 0 & -0.33 & -0.33 & -1 & 0.33 & 0 \\
\hline \multicolumn{2}{|c|}{$\mathrm{z}_{\mathrm{j}}$} & 2 & 3 & -0.67 & 0 & 0 & -0.33 & 0.67 & 0 & 0.33 & \\
\hline \multicolumn{2}{|c|}{$\mathrm{c}_{\mathrm{j}}-\mathrm{z}_{\mathrm{i}}$} & $\mathbf{0}$ & 0 & 0.67 & 0 & 0 & 0.33 & $\mathrm{M}-0.67$ & $\mathrm{M}$ & $\mathrm{M}-0.33$ & \\
\hline
\end{tabular}

From tab. 2, the matrix columns marked in gray are read $\boldsymbol{B}^{-1}$ inverse to the matrix of coefficient vectors for the variables $x_{1}, x_{2}, x_{5}, x_{4}$. In the case of the model $\boldsymbol{M 1}$ the matrix $B^{-1}$ consists of columns corresponding to the other variables $x_{7}, x_{8}, x_{5}, x_{9}$ (the first model base $\boldsymbol{M 1}$ ). In the last column, tab. 2 in lines corresponding to variables $x_{4} . x_{5}$, which are not decision variables, correspond to the zero value in the optimal solution. This means that starting from equality $\boldsymbol{B}^{-1} \cdot \boldsymbol{b}=\left[x_{1}^{*} ; x_{2}^{*} ; x_{5}^{*} ; x_{4}^{*}\right]^{T}=[120 ; 80 ; 0 ; 0]^{T}$ two highlighted vectors are obtained $\left(v=\left[-5 \frac{1}{3} ; 0 ; 1 ; \frac{1}{3}\right]^{T}\right.$ and $\boldsymbol{w}=\left[-\frac{1}{3} ;-1 ; 0 ; \frac{1}{a}\right]^{T}$ ) orthogonal to the vector $\boldsymbol{b}$. These vectors together with a single solution $y_{0}^{*}$ are enough to open the 2 -dimensional space $\mathcal{H}$ containing a set $P$. $P \subset \mathcal{H}=\left\{\boldsymbol{y} \in \mathbb{R}^{4}: \boldsymbol{y}=\boldsymbol{y}_{0}^{*}+s \cdot v+t \cdot \boldsymbol{w}, s, t \in \mathbb{R}\right\}$.

Hence, in order to determine the exact set $P$, on account of $s, t$ the inequality system resulting from the boundary restrictions of the dual model $\boldsymbol{D} \_\boldsymbol{M 1}$ [16, p. 104] should be solved:

$$
y=\left[\begin{array}{l}
1 \\
1 \\
0 \\
0
\end{array}\right]+s \cdot\left[\begin{array}{c}
-16 / 3 \\
0 \\
1 \\
1 / 3
\end{array}\right]+t \cdot\left[\begin{array}{c}
-1 / 3 \\
-1 \\
0 \\
1 / 3
\end{array}\right] \geq\left[\begin{array}{l}
0 \\
\geq \\
0 \\
0 \\
0
\end{array}\right] \text {. (1) }
$$

Choosing a pair of intersecting straight lines from the system (1) we obtained permutationally $4 ! /(2 ! \cdot 2 !)=6$ argument pairs $s, t \in \mathbb{R}:(0 ; 0), \quad(-1 ; 1),(0 ; 1)$, $(0,2 ;-0,2),(0 ; 3),(1 / 8 ; 1)$. A graphic illustration of the system solution (1) is the darkest area in Fig. 1.

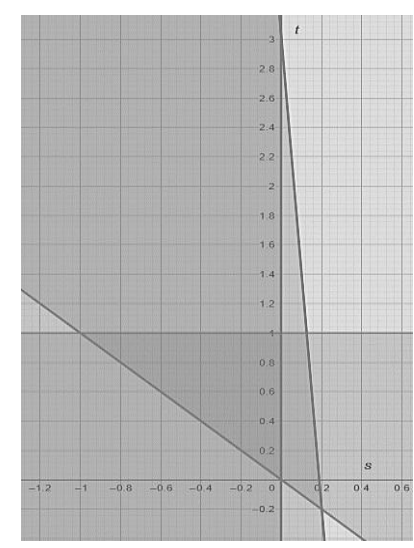

Fig. 1. The darkest area denotes a set of system solutions (1).

The intersections of straight pairs from Fig. 1 are vectors: $\quad y_{0}^{*}=[1 ; 1 ; 0 ; 0]^{T}, \quad y_{1}^{*}=[6 ; 0 ;-1 ; 0]^{T}$, $y_{2}^{*}=[2 / 3 ; 0 ; 0 ; 1 / 3]^{T}, \quad y_{3}^{*}=[0 ; 1,2 ; 0,2 ; 0]^{T}$, $y_{4}^{*}=[0 ;-2 ; 0 ; 1]^{T}, \quad y_{5}^{*}=[0 ; 0 ; 1 / 8 ; 3 / 8]^{T}$. The first three of them: $\boldsymbol{y}_{0}^{*}, \boldsymbol{y}_{1}^{*}, \boldsymbol{y}_{2}^{*}$, satisfy the system (1) and only they constitute base vectors belonging to $P$.

In the case of increasing the demand for water intake by one unit, the obtained dual price vectors $\boldsymbol{y}_{0}^{*}, \boldsymbol{y}_{1}^{*}, \boldsymbol{y}_{2}^{*}$ indicate that the costs incurred by the system may increase accordingly by: 1 or 6 or $2 / 3$ monetary units. Thus, for different change vectors $\Delta_{\mathrm{j}} \boldsymbol{b}=\left[1, \Delta_{\mathrm{j}} b_{2}, \Delta_{\mathrm{j}} b_{3}, \Delta_{\mathrm{j}} b_{4}\right]$ of the right-hand sides of $C O$ these three situations of change in the total costs are highlighted 


$$
\Delta_{\mathrm{j}} O F\left(\Delta b_{1}=1\right)=\Delta_{\mathrm{j}} \boldsymbol{b}^{T} \cdot \boldsymbol{y}_{j}^{*}=\left\{\begin{array}{c}
1 \text { for } j=0 \\
6 \text { for } j=1 \\
2 / 3 \text { for } j=2
\end{array} .\right.
$$

This is different than in the case of standard postoptimization procedures dealing with disjoint changes $[17$, p. 59], i.e. the sensitivity analysis where dual prices are related to changes in only single the parameters of the right-hand sides of $C O$. In the considered example, different change vectors should be determined on the basis of various dual variables $\Delta_{\mathrm{j}} \boldsymbol{b}$. But in order for the further procedure to become somewhat similar to the standard sensitivity analysis, the components of the vector should be selected in a specific way $\Delta_{\mathrm{j}} \boldsymbol{b}$. For this purposes, changes have been made $\Delta_{\mathrm{j}} \boldsymbol{b}$ orthogonal to vectors $\boldsymbol{v}$ and $\boldsymbol{w}$ or changes in the form of $\Delta_{\mathrm{j}} \boldsymbol{b} \cdot \boldsymbol{v}=0=\Delta_{\mathrm{j}} \boldsymbol{b} \cdot \boldsymbol{w}$. This means that with a unit increase in water demand (CO1) the bonds are retained in the remaining $C O$. In addition, due to the location of non-zero elements in vectors $y_{0}^{*}, \boldsymbol{y}_{1}^{*}, \boldsymbol{y}_{2}^{*}$ for the change in the value of the objective function to result from the dual price corresponding only to $\Delta b_{1}=1$ similarly as in the case of disjoint changes, then in vectors $\Delta_{\mathrm{j}} \boldsymbol{b}$ some elements can take the value of zero resulting in: $\Delta_{\mathrm{j}} \boldsymbol{b}=\left[1, \Delta_{\mathrm{j}} b_{2}, \Delta_{\mathrm{j}} b_{3}, \Delta_{\mathrm{j}} b_{4}\right]$ where $\Delta_{\mathrm{j}} b_{\mathrm{j}+2}=0$ for $j=0,1,2$. And the orthogonality conditions alone take the form of three systems of equations:

$$
\left\{\begin{array}{c}
-16 / 3 \cdot 1+0+\Delta_{\mathrm{j}} b_{3}+1 / 3 \Delta_{\mathrm{j}} b_{4}=0 \\
-1 / 3 \cdot 1-\Delta_{\mathrm{j}} b_{2}+0+1 / 3 \Delta_{\mathrm{j}} b_{4}=0 . \\
\Delta_{\mathrm{j}} b_{\mathrm{j}+2}=0
\end{array}\right.
$$

Solutions (2) take the form: $\Delta_{0} b=[1,0,5,1]^{T}$, $\Delta_{1} b=[1,5,0,16]^{T}, \Delta_{2} b=[1,-1 / 3,16 / 3,0]^{T}$. These solutions have different interpretations: a unitary increase in water demand should be accompanied at $j=0$ by an increase in the value of right-hand sides of $C O 3$ by 5 and $C O 4$ by 1 unit; at $j=1$ by an increase in the value of the right-hand sides in $\mathrm{CO} 2$ by 5 and $\mathrm{CO} 4$ by 16 units; at $j=2$ by a drop in the value of the righthand side in $\mathrm{CO} 2$ by $1 / 3$ and an increase in $\mathrm{CO} 3$ by $16 / 3$ units. Because $C O$ refer to the principle of the inviolability of water flow in the river in the "S" water gauge cross-section, as well as to the technical reasons of the dependence between intakes, thus forcing these changes to the right-hand sides of $C O$ can sometimes be too expensive or unattainable due to environmental reasons. What is more for the unit increase of the righthand side of $C O 1$ also the left-hand sides of $\mathrm{CO}$ for $j=0,1,2$ yield an adjusted optimal solution in such a corrected model M1 sometimes going beyond the set of solutions for an acceptable initial model $\boldsymbol{M 1}$, and yes:

1. change $\Delta_{0} b=[1,0,5,1]^{T}$ causes the left-hand sides of $\mathrm{CO}$ to extend beyond $\mathrm{CO} 3$ of the model $\mathrm{M1}$.

2. change $\Delta_{1} b=[1,5,0,16]^{T}$ it does not cause the lefthand sides of $C O$ to extend beyond $C O$ of the model M1.

3. change $\Delta_{2} b=[1,-1 / 3,16 / 3,0]^{T}$ causes the lefthand sides of $\mathrm{CO}$ to extend beyond $\mathrm{CO} 2, \mathrm{CO} 3$ of the model $\boldsymbol{M 1}$.
Thus, due to the degeneration of the model with each of the indicated costs, there is a different way of reconstruction of the water intake system and so the change $\Delta_{1} b$ does not force exceeding the norms previously set out in the model $\boldsymbol{M 1}$, but it is the most expensive. While changes $\Delta_{0} b$ and $\Delta_{2} b$ turn out to be much cheaper, each time they require changes that deviate from the previously established norms. Therefore, ensuring increased water consumption in addition to considering the problems of meeting additional investment costs requires the introduction of skillful changes related to the water intake conditions.

\section{Conclusions}

1. Linear water management models should enable postoptimization also in degenerate cases, which have not yet found sufficient managerial and mathematical analysis in literature [3-10]. So far, it has been problematic to generate all the nodal points of an infinite set $P$ [13]. This work has solved this problem and additionally has provided an appropriate interpretation of the obtained results.

2. Managerial decisions require a review of all nodal solutions to the dual model $\boldsymbol{D} \_\boldsymbol{M} \mathbf{1}$ and any associated changes to many vector parameters associated with them $\boldsymbol{b}$. It may turn out that some such changes are cheaper (here $\Delta_{0} b$ and $\Delta_{2} b$ ) but are difficult to implement as they may require technical adjustments or changes to previously adopted hydrological norms.

3. vectors $\boldsymbol{v}$ and $\boldsymbol{w}$ read from the matrix $\boldsymbol{B}^{-1}$ based on the last simplex table (tab. 2) were used to construct a) the entire 2-dimensional space $\mathcal{H}$ containing the set $P$ of infinitely many solutions to the dual model, b) such solutions $\boldsymbol{y}_{j}^{*}$ to the dual model, which are only the nodal points of the set $P$ and related c) vectors $\Delta_{\mathrm{j}} b$ corresponding to the unit parameter change $\Delta b_{1}$ in vector $\boldsymbol{b}$.

\section{References}

1. J. Połomska, W. Chmielowski, Annals of Warsaw University of Life Sciences - SGGW Land Reclamation 44(1), 15-24 (2012)

2. J. Wawrzosek, S. Ignaciuk, Logistyka 1, 425-431 (2016)

3. M. Akgul, JORS 35(5), 425-431 (1984)

4. H. Arsham, JORS 43(6), 121-139 (1992)

5. T. Gal, Postoptimal analysis, Parametric

Programming and Related Topics: Degeneracy, Multicriteria Decision Making, Redundancy

(McGraw-Hill, New York, 1986)

6. T. Gal, OR Spektrum, 8(2), 59-71 (1986)

7. H. J. Greenberg, NRL 33(4), 635-655 (1986)

8. B. Jansen, J. J.de Jong, C. Roos, T. Terlaky, European JORS 101(1), 15-28 (1997) 
9. T. Koltai, T. Terlaky, Int. J. Prod. Econ. 65(3), 257$274(2000)$

10. T. Koltai, V. Tatay, Int. J. Prod. Econ. 131(1), 392398 (2011)

11. S. Ignaciuk, Post-optimization procedures for the selected transport issues in agricultural engineering $(\mathrm{PhD}$ thesis, Department of Applied Mathematics and Computer Science, Faculty of Production Engineering, University of Life Sciences in Lublin, 2018)

12. J. Wawrzosek, S. Ignaciuk, Logistyka 4, 8473-8481 (2015)

13. J. Wawrzosek, S. Ignaciuk, Eksploatacja i Niezawodnosc-Maintenance and Reliability (2018) (in review)

14. Y.-L. Chang, K. Desai, T. Kratzer, WinQSB v. 2.0., Decision Support Software for MS/OM (John Wiley \& Sons, New Jersey, 2003)

15. G. B. Dantzig, M. N. Thapa, Linear Programming 1: Introduction. Linear programming 1: Introduction (Springer - Verlag, 1997)

16. R. Sioshansi, A. J. Conejo, Optimization in Engineering: Models and Algorithms (Springer, 2017)

17. A. Busłowski, Stabilność rozwiazania optymalnego zadania programowania liniowego (Wydawnictwo Uniwersytetu w Białymstoku, Białystok, 2000) 